\title{
Evaluation in mice of Brucella ovis attenuated mutants for use as live vaccines against $B$. ovis infection
}

\author{
Pilar Sancho ${ }^{1}$, Carmen Tejedor ${ }^{1}$, Rebeca S Sidhu-Muñoz ${ }^{1,2}$, Luis Fernández-Lago ${ }^{1,2}$ and Nieves Vizcaíno ${ }^{1,2^{*}}$
}

\begin{abstract}
Brucella ovis causes ram contagious epididymitis, a disease for which a specific vaccine is lacking. Attenuated Brucella melitensis Rev 1, used as vaccine against ovine and caprine brucellosis caused by B. melitensis, is also considered the best vaccine available for the prophylaxis of $B$. ovis infection, but its use for this purpose has serious drawbacks. In this work, two previously characterized B. ovis attenuated mutants ( $\Delta$ omp25d and $\Delta$ omp22) were evaluated in mice, in comparison with $B$. melitensis Rev 1 , as vaccines against $B$. ovis. Similarities, but also significant differences, were found regarding the immune response induced by the three vaccines. Mice vaccinated with the $B$. ovis mutants developed anti-B. ovis antibodies in serum of the $\operatorname{lgG}_{1}, \lg _{2 a}$ and $\lg _{2 b}$ subclasses and their levels were higher than those observed in Rev 1-vaccinated mice. After an antigen stimulus with B. ovis cells, splenocytes obtained from all vaccinated mice secreted similar levels of TNF- $a$ and IL12(p40) and remarkably high amounts of IFN- $\gamma$, a crucial cytokine in protective immunity against other Brucella species. By contrast, IL-1a -an enhancer of T cell responses to antigen- was present at higher levels in mice vaccinated with the $B$. ovis mutants, while IL-10, an anti-inflammatory cytokine, was significantly more abundant in Rev 1-vaccinated mice. Additionally, the B. ovis mutants showed appropriate persistence, limited splenomegaly and protective efficacy against B. ovis similar to that observed with B. melitensis Rev 1 . These characteristics encourage their evaluation in the natural host as homologous vaccines for the specific prophylaxis of B. ovis infection.
\end{abstract}

\section{Introduction}

Brucellosis caused by Brucella ovis mainly induces chronic epididymitis and orchitis in rams. The disease is widely distributed and constitutes one of the most important causes of reproductive failure in sheep [1]. In addition to a reduction in male fertility, B. ovis induces occasional abortions in ewes and increases the risk of perinatal mortality and low weight in lambs, thus having an important economic impact in the livestock sector [1].

Brucella strains are smooth (S) or rough (R), depending on the presence or absence of O-polysaccharide (O-PS) chains in the lipopolysaccharide (LPS) respectively. Other outer membrane $(\mathrm{OM})$ components -such as OM proteins (OMPs) or the core and lipid A of LPS- are masked by O-PS in S strains, while in R brucellae, such as B. ovis, they

\footnotetext{
* Correspondence: vizcaino@usal.es

'Departamento de Microbiología y Genética, Edificio Departamental, Universidad de Salamanca, Plaza Doctores de la Reina s/n, 37007 Salamanca, Spain

${ }^{2}$ Instituto de Investigación Biomédica de Salamanca (IBSAL), Hospital Universitario de Salamanca, Po de San Vicente 52-182, 37007 Salamanca, Spain
}

are exposed on the bacterial surface [2]. In infected animals and humans, the O-PS chains induce a strong antibody response that constitutes the basis for the serological diagnosis of infections caused by S Brucella strains [2].

Brucella melitensis Rev 1 is an S live attenuated strain currently used for vaccination campaigns against ovine and caprine brucellosis, which is almost exclusively caused by $B$. melitensis and B. ovis. It is also considered the best vaccine available for the prophylaxis of $B$. ovis infection despite being mainly used for preventing $B$. melitensis infection [3-7]. However, several drawbacks are associated with Rev 1 vaccine, such as its variable efficacy, the induction of antibodies against S-LPS interfering with the diagnosis of brucellosis caused by B. melitensis and other $\mathrm{S}$ brucellae, the ability to induce human infections and abortions in animals and its resistance to streptomycin $[5,8,9]$. Additionally, due to its serological cross-reactivity with other S Brucella strains, it is banned in countries where B. melitensis has been eradicated, some of which are 
important sheep producers and where B. ovis infection constitute a serious problem. Therefore, the development of a new vaccine able to substitute $B$. melitensis $\operatorname{Rev} 1$ strain in the prophylaxis of $B$. ovis infection is a matter of great interest.

Subcellular vaccines based on the hot saline extract (HS) of B. ovis -mainly composed of R-LPS and OMPshave been extensively analyzed in mice and rams but their protective activity is limited [10-14]. Among these vaccines, HS encapsulated in microparticles has provided the best results in terms of protection [12]. R-LPS and/ or recombinant proteins, either as purified antigens or as DNA vaccines requiring repeated inoculations, have also been assayed, with different success rates [13-17]. Considering that the best vaccines available against brucellosis caused by S Brucella strains are homologous $S$ live attenuated strains [18], the development of an $\mathrm{R}$ $B$. ovis attenuated vaccine strain seems to be an interesting approach for the control of $B$. ovis infection.

In light of the above, the aim of this work was to evaluate the immunogenicity and protective activity against B. ovis infection of two B. ovis attenuated mutants obtained previously by inactivation of the genes coding for Omp25d and Omp22 [19], two OMPs of the Omp25/Omp31 family $[20,21]$. Considering that routine analysis of Brucella vaccines in the natural host is precluded due to economical and practical difficulties [22], the murine model was selected for this purpose since it is proposed by the World Organisation for Animal Health (OIE) as a method for the evaluation of Brucella vaccines administered in sheep [23] and it is currently used in brucellosis research for the assessment of the protective activity of vaccines [22,24]. Additionally, a good correlation between the mouse model and sheep has been observed for other Brucella vaccines [25] and the B. ovis attenuated mutants have been analyzed in parallel with $B$. melitensis Rev1 -the attenuated strain used in official vaccination campaigns against sheep brucellosis [3,23]- that constitutes a control of recognized vaccine efficacy in the natural host.

\section{Materials and methods}

\section{Bacterial strains and culture conditions}

The Brucella strains used in this work are shown in Table 1. The R virulent B. ovis PA strain and smooth (S) attenuated B. melitensis Rev 1 classical vaccine were obtained from the
Institut National de la Recherche Agronomique, Nouzilly, France. Attenuated B. ovis PNV25dA ( $\Delta o m p 25 d)$ and B. ovis PNV22A ( $\triangle$ omp22) are mutant strains derived from virulent $B$. ovis PA [19]. They were obtained previously by substitution of omp25d or omp22, respectively, by a kanamycin (Kan) resistance gene [19].

All Brucella strains were propagated in tryptic soy agar (Pronadisa-Laboratorios Conda, Torrejón de Ardoz, Spain) supplemented with $0.3 \%$ yeast extract (Pronadisa-Laboratorios Conda) and 5\% horse serum (Gibco-Life Technologies, Grand Island, USA) (TSA-YE-HS). When appropriate, depending on the resistance profile of each Brucella strain (Table 1), streptomycin (Strep) or Kan (Sigma-Aldrich, St. Louis, USA) was added to TSA-YE-HS medium to a final concentration of $50 \mu \mathrm{g} / \mathrm{mL}$. Bacteria were incubated at $37^{\circ} \mathrm{C}$ in a $5 \% \mathrm{CO}_{2}$ atmosphere.

\section{Mice and inoculation procedure}

Female 6-week-old BALB/c mice (Charles River Laboratories, Chatillon-sur-Chalaronne, France), received at our laboratory one week previously, were used. They were randomly distributed into experimental groups and kept with water and food ad libitum in the animal experimentation facilities of the University of Salamanca (registration number PAE SA-001). Procedures with mice were designed according to Spanish and European legislation regarding the use of animals in research (RD 1201/05 and directive 2010/63/UE).

Vaccination and challenge with Brucella strains were performed by intraperitoneal inoculation of bacterial suspensions in phosphate-buffered saline (PBS; $\mathrm{pH}$ 7.2) prepared from fresh cultures incubated for $44 \mathrm{~h}$. The number of colony-forming units per $\mathrm{mL}(\mathrm{CFU} / \mathrm{mL})$ was estimated by optical density (OD) readings at $600 \mathrm{~nm}$ $\left(\mathrm{OD}_{600}\right)$ and the exact doses administered were determined retrospectively by triplicate plating of the properly diluted inocula on TSA-YE-HS.

\section{Immunization of mice and sample collection for evaluation} of the immune response and vaccine strain persistence Mice were inoculated with PBS (unvaccinated control), with the $B$. melitensis Rev 1 classical vaccine $\left(10^{5} \mathrm{CFU}\right)$, or with the $\Delta o m p 25 d\left(10^{7} \mathrm{CFU}\right)$ or $\Delta o m p 22\left(10^{8} \mathrm{CFU}\right)$ mutants of $B$. ovis PA. The dose of vaccination for $B$. melitensis Rev 1 was that commonly used for vaccine

Table 1 Brucella strains used in this work

\begin{tabular}{|c|c|c|c|c|}
\hline Strain & Origin $^{a}$ & Virulence (relevant genotype) & LPS phenotype & Relevant antibiotic resistance \\
\hline B. melitensis Rev 1 & BCCN V4a & Classical attenuated vaccine & Smooth & Streptomycin \\
\hline B. ovis PA & BCCN 76-250 & Virulent strain & Rough & - \\
\hline B. ovis PNV25dA & {$[19]$} & Recombinant B. ovis PA attenuated mutant ( $\Delta$ omp25d) & Rough & Kanamycin \\
\hline B. ovis PNV22A & [19] & Recombinant B. ovis PA attenuated mutant ( $\Delta$ omp22) & Rough & Kanamycin \\
\hline
\end{tabular}

${ }^{\mathrm{a} B C C N}$, Brucella Culture Collection, Nouzilly, France. 
studies in mice [22], while the vaccination doses for the $B$. ovis attenuated mutants were selected according to previous results of spleen colonization when they were inoculated at a dose of $5 \times 10^{6} \mathrm{CFU} / \mathrm{mice}$ [19] and taking into account vaccine studies with other Brucella species [22]. Thus, considering that the $\Delta o m p 22$ mutant was severely attenuated in that previous study [19], the dose of $10^{8} \mathrm{CFU} /$ mice was selected to analyze its vaccine properties, while vaccination with the $\Delta o m p 25 d$ mutant -that showed a reduced persistence but a high level of spleen colonization at week 1 post-inoculation (pi) (about $2 \log$ units higher than the parental strain) [19]- was performed at a dose of $10^{7} \mathrm{CFU} /$ mice.

Sera for analysis of the antibody response were obtained from submandibular blood extracted from five mice per group at selected time-points. The same mice were then euthanized and their spleens isolated in order to determine both the CFU number of the vaccine strain (persistence assay) and the cytokine profile.

To evaluate the ability of splenocytes from vaccinated mice to secrete cytokines in response to a stimulus with heat-inactivated $B$. ovis PA whole cells, five mice per group were immunized as described above and their spleens were removed 28 days later for further ex vivo analysis of spleen cell cultures, as detailed below.

\section{Evaluation of vaccine strain persistence}

Persistence of the vaccine strains in mice was evaluated by determining the bacterial CFU in spleen at $1,3,7,14$, 21, 28 and 49 days post-inoculation (dpi). At each selected pi time-point, spleens from five mice per group were weighed and individually homogenized in 9 vol of Hanks' balanced solution (Gibco-Life Technologies). Ten-fold serial dilutions were plated onto TSA-YE-HS to determine bacterial counts. The results were expressed as means \pm SD $(n=5)$ of the log CFU/spleen for each strain. For each vaccination group, the mean $\pm \operatorname{SD}(n=5)$ of the spleen weight in grams was also calculated.

\section{Evaluation of the antibody response in serum against whole cells of $B$. ovis}

Serum immunoglobulin (Ig) G (IgG) titers in immunized mice were determined by indirect enzyme-linked immunosorbent assay (i-ELISA) at 1, 3, 7, 14, 21, and 28 dpi (pre-challenge period in protection experiments). Plates (96-well Maxisorp, Nunc-Thermo Fisher Scientific, Roskilde, Denmark) were coated by overnight incubation with $100 \mu \mathrm{L}$ of $B$. ovis PA suspensions $\left(\mathrm{OD}_{600}=1\right)$ prepared in PBS from heat-inactivated $\left(1 \mathrm{~h}, 65{ }^{\circ} \mathrm{C}\right)$ fresh cultures (44 $\mathrm{h}$ of growth). The wells were then incubated sequentially with $5 \%$ skim milk in PBS $\left(30\right.$ min at $\left.37{ }^{\circ} \mathrm{C}\right)$, double-serial dilutions of mouse sera in PBS containing $0.05 \%$ Tween 20 (PBS-T; $1 \mathrm{~h}$ at $37{ }^{\circ} \mathrm{C}$ ) and goat antimouse IgG (Fc)-peroxidase conjugate (Sigma-Aldrich) diluted 1:4000 in PBS-T (1 $\mathrm{h}$ at room temperature). The different steps were separated by three washes with $0.9 \%$ $\mathrm{NaCl}, 0.05 \%$ Tween 20 . Finally, the substrate solution -1 mM 2,2'-azino-di-(3-3-ethylbenzothiazoline-sulfonic acid) (ABTS; Sigma-Aldrich) and $2 \mathrm{mM} \mathrm{H}_{2} \mathrm{O}_{2}$ (Sigma-Aldrich) in $0.1 \mathrm{M}$ citrate, $\mathrm{pH} 4.2$ - was added to the wells and the $\mathrm{OD}_{405}$ readings were recorded on a Microplate Reader 550 (Bio-Rad, Hercules, USA) after $30 \mathrm{~min}$ incubation at room temperature. IgG isotypes were determined under the same conditions but using goat anti-mouse $\mathrm{IgG}_{1^{-}}, \operatorname{IgG}_{2 \mathrm{a}^{-}}$or $\operatorname{IgG}_{2 \mathrm{~b}}$-peroxidase conjugates (Santa Cruz Biotechnology, Dallas, USA).

Antibody titers in serum were defined as the inverse of the highest serum dilution scoring an $\mathrm{OD}_{405}$ value two times higher than that obtained with the blank (mean $\mathrm{OD}_{405}$ of six wells in which serum was replaced by dilution buffer). The results for IgG were represented as means \pm SD of the log of the titers obtained with five mice analyzed individually. The results concerning IgG isotypes were represented as means $\pm \mathrm{SD}$ of the $\mathrm{OD}_{405}$ values obtained with a 1/100 dilution of a pool of the five sera assayed in triplicate.

\section{Evaluation of cytokines in spleen and in splenocyte cultures}

Cytokine levels in the spleens of immunized mice were evaluated at $1,3,7,14,21$ and 28 dpi (pre-challenge period in protection experiments). CHAPS (Sigma-Aldrich) was added ( $1 \%$ final concentration) to mouse spleens homogenized in Hanks balanced salt solution (GibcoLife Technologies) (see vaccine persistence section) [26,27]. After a 1-h incubation at $4{ }^{\circ} \mathrm{C}$, cell debris was removed by centrifugation and the supernatants were stored at $-80{ }^{\circ} \mathrm{C}$ until analysis. The levels of interferon- $\gamma$ (IFN- $\gamma$ ), tumor necrosis factor- $\alpha$ (TNF- $\alpha$ ), interleukin- $1 \alpha$ (IL- $1 \alpha)$, IL-10 and IL-12(p40) were quantified by sandwich ELISA with OptEIA $^{\mathrm{TM}}$ Mouse Sets specific to each cytokine, as instructed by the manufacturer (BD Biosciences, San Diego, USA) and described elsewhere [28]. The results for each vaccination group were expressed as means \pm SD of the cytokine quantity (ng) detected in the spleens of five individual mice at each time-point.

Cytokine production was also evaluated, after stimulation with heat-inactivated $B$. ovis PA whole cells, in cell cultures of splenocytes obtained from immunized mice at $28 \mathrm{dpi}$. Uniform single-cell suspensions were prepared by gentle disruption of spleens, as described previously [28]. Cells were suspended in complete RPMI medium (RPMI supplemented with $10 \%$ fetal calf serum, $4 \mathrm{mM}$ L-glutamine, $1 \mathrm{mM}$ sodium pyruvate, $0.05 \mathrm{mM} 2$-mercaptoethanol (Gibco-Life Technologies) and $100 \mu \mathrm{g} / \mathrm{mL}$ gentamicin), distributed in 24-well plates $\left(4 \times 10^{5}\right.$ cells/ well in $1 \mathrm{~mL}$ ) and cultured at $37^{\circ} \mathrm{C}$ and a $5 \% \mathrm{CO}_{2}$ atmosphere. Splenocytes were stimulated by exposure to heatinactivated $\left(1 \mathrm{~h}\right.$ at $\left.65^{\circ} \mathrm{C}\right)$ B. ovis PA whole cells $\left(10^{7} \mathrm{CFU} /\right.$ 
well), $10 \mu \mathrm{g} / \mathrm{mL}$ of the mitogen concanavalin A (SigmaAldrich) as a positive control of cell proliferation, or culture medium as a negative control. The stimulating agents were prepared in complete RPMI medium and added to each well in $100-\mu \mathrm{L}$ volumes. After $72 \mathrm{~h}$, the supernatants were harvested, centrifuged and stored at $-80{ }^{\circ} \mathrm{C}$ until use for cytokine quantification, performed as described above. Two wells were used for each experimental condition and mouse. The results for each vaccination group were expressed as means \pm SD of the cytokine amount (ng/well) in the supernatants of splenocytes obtained from five individual mice. The results obtained with the positive and negative controls (concanavalin A and RPMI as stimulating agents, respectively) were as expected and are not shown.

\section{Protection studies in mice}

Mice inoculated with PBS (unvaccinated control), $B$. melitensis Rev 1 ( $\left.10^{5} \mathrm{CFU} / \mathrm{mouse}\right)$, B. ovis $\Delta$ omp $25 d$ $\left(10^{7} \mathrm{CFU} / \mathrm{mouse}\right)$ or B. ovis $\Delta$ omp $22\left(10^{8} \mathrm{CFU} / \mathrm{mouse}\right)$ were challenged 28 days later with $10^{5} \mathrm{CFU}$ of virulent B. ovis PA. Three weeks after the challenge (49 days after immunization), the bacterial CFU numbers in spleen were counted in five mice from each experimental group, as described previously [19]. The CFU number of virulent B. ovis PA in mice vaccinated with $B$. melitensis $\operatorname{Rev} 1$ (Strep-resistant strain; Table 1) was obtained by subtracting the CFU obtained in TSA-YE-HS-Strep medium from those obtained in the same medium without antibiotic. Similarly, differential counts in medium with and without Kan were used to determine the CFU of the challenge strain in the spleens of mice vaccinated with the B. ovis attenuated strains. Results were expressed as means \pm SD $(n=5)$ of the $\log \mathrm{CFU} /$ spleen of $B$. ovis PA for each vaccination group.

\section{Statistical analyses}

Statistical comparisons between means were performed using analysis of variance. The levels of significance of the differences between the experimental groups were determined with the post-hoc Fisher's protected least significant differences (PLSD) test.

\section{Results}

\section{Persistence of vaccine strains in spleen}

Spleen weight and bacterial counts were determined at several pi time-points in mice inoculated with $B$. melitensis Rev 1, B. ovis $\Delta$ omp25d, and B. ovis $\Delta o m p 22$ (retrospectively determined doses of $0.9 \times 10^{5}, 1.2 \times 10^{7}$, and $0.80 \times 10^{8} \mathrm{CFU} /$ mouse, respectively).

Under the assay conditions, spleen colonization by $B$. melitensis Rev1 and B. ovis $\Delta o m p 25 d$ was quite similar, with a peak of about $7 \log \mathrm{CFU} /$ spleen at 1 week pi and a progressive decrease in bacterial counts thereafter until the end of the experiment on day 49 pi (Figure 1A). However, while the classic $B$. melitensis Rev 1 vaccine was detected in most of the animals on days 28 and 49 pi, all mice inoculated with the B. ovis $\Delta$ omp $25 d$ mutant had cleared the infection by day 49 pi (according to the detection limit of the technique) and only 1 out 5 five mice showed bacteria in its spleen at day $28 \mathrm{pi}$. With the B. ovis $\Delta o m p 22$ attenuated mutant, the highest level of spleen colonization extended from day 3 to day 21 pi and was $2 \log$ units lower (about $5 \log \mathrm{CFU} /$ spleen) than that obtained with the two other vaccines on day 7 pi. Thereafter, B. ovis $\Delta o m p 22$ behaved similarly to the other vaccines (Figure $1 \mathrm{~A})$.

Regarding spleen weight (Figure 1B), the two B. ovis attenuated mutants did not induce significant inflammation of the spleen along the assay, while the B. melitensis Rev 1 classic vaccine induced a strong transient splenomegaly, reaching its highest level at $14 \mathrm{dpi}$. At that moment, spleen weight in the mice inoculated with $B$. melitensis Rev 1 was about 4 times higher than that observed in mice inoculated with the $B$. ovis mutants (Figure 1B).

\section{Antibody response against $B$. ovis induced by the vaccines}

The levels of serum IgG able to react with whole $B$. ovis cells in i-ELISA were evaluated up to day 28 pi (prechallenge period in the protection experiments) in mice inoculated, as described for the persistence assay, with PBS (negative control group) or each of the three attenuated vaccines.

In mice immunized with the two $B$. ovis attenuated mutants, the IgG response against whole B. ovis cells became evident by day 7 pi and increased progressively thereafter until the end of the experiment (Figure 2A). On days 7 and 14 pi, IgG titers were higher in mice immunized with $B$. ovis $\Delta o m p 22$ than in those immunized with $B$. ovis $\Delta o m p 25 d$, but they reached similar levels by day 28 pi (Figure 2A). In comparison, the IgG response in mice immunized with $B$. melitensis Rev 1 was delayed and weaker, since detectable titers appeared on day $14 \mathrm{pi}$ and were lower than those observed with the $B$. ovis vaccines at all time-points checked (Figure 2A). Inside each vaccination group, no important differences were detected in the proportion of the $\operatorname{IgG}_{1}, \operatorname{IgG}_{2 \mathrm{a}}$ and $\operatorname{IgG}_{2 \mathrm{~b}}$ isotypes (Figure 2B-D).

\section{Spleen cytokines in vaccinated mice}

The cytokine profile in spleen was also evaluated until day 28 pi in mice inoculated, as described for the persistence assay, either with PBS or with the attenuated strains.

The highest levels of IFN- $\gamma$, TNF- $\alpha$, IL- $1 \alpha$, IL-10 and IL-12(p40) in spleen were detected in mice immunized with $B$. melitensis Rev 1 (Figure 3). In this group of mice, 

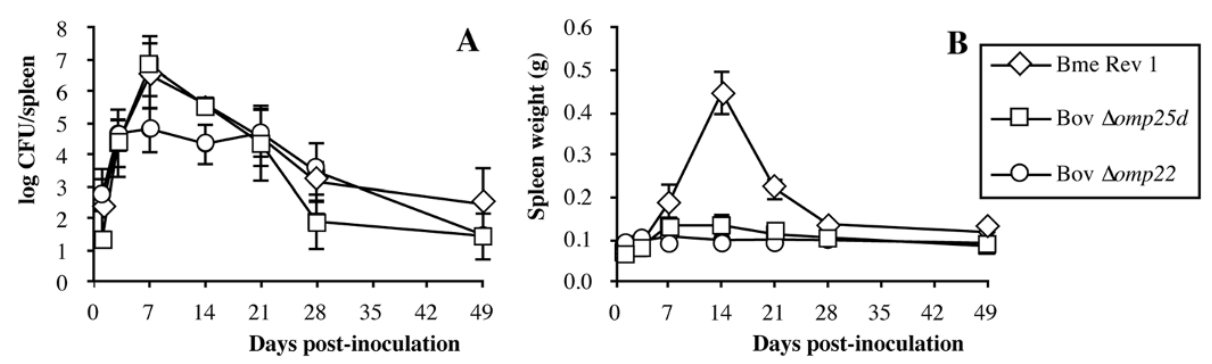

Figure 1 Kinetics of splenic infection and spleen weight in mice inoculated with the vaccines. $B$. melitensis Rev 1 (Bme Rev $1 ; 1 \times 10^{5} \mathrm{CFU} /$ mouse), B. ovis PNV25dA (Bov $\Delta$ omp25d; $1 \times 10^{7}$ CFU/mouse), and B. ovis PNV22A (Bov $\Delta$ omp22; $1 \times 10^{8}$ CFU/mouse) were used as vaccines. Results are expressed as means \pm SD $(n=5)$ of the log CFU/spleen (A) or spleen weight (B) at each time-point.

all cytokines, except IL-10, were present in high levels on days 7 and $14 \mathrm{pi}$, with a production peak on day 14 pi, and a decrease thereafter until day 28 pi, when all cytokines had levels similar to those detected in mice inoculated with PBS (Figure 3). IL-10 production in the Rev 1-vaccinated mice followed a similar pattern but with a delay of 7 days, the highest levels being detected on days 14 and 21 pi (Figure 3D). Whereas TNF- $\alpha$ and IL-10 scored the lowest levels (about 1.5 and $2 \mathrm{ng} /$ spleen, respectively), the strongest response was obtained with IL-1 $\alpha$ and IL-12(p40) (maximum levels about $10 \mathrm{ng}$ / spleen) followed by IFN- $\gamma$ ( $5 \mathrm{ng} / \mathrm{spleen})$.

Although to a lesser extent than B. melitensis Rev 1, B. ovis $\Delta$ omp $25 d$ also induced the production in spleen of all cytokines tested (Figure 3), with the exception of IL-10 (Figure 3D). A peak of cytokine production was observed on day $7 \mathrm{pi}$, which was followed by a progressive decrease until day 28 pi, when no differences between the mice inoculated with B. ovis $\Delta o m p 25 d$ and the PBSinoculated mice were observed (Figure 3 ).

In general, the mice immunized with B. ovis $\Delta$ omp 22 did not show statistically significant differences in cytokine production in spleen when compared with mice inoculated with PBS (Figure 3). The exception was IL-12 (p40) on day $7 \mathrm{pi}$, whose concentration in spleen was slightly higher in mice inoculated with B. ovis $\Delta o m p 22$ (Figure 3E).

\section{Cytokine secretion by splenocytes of vaccinated animals} in response to a stimulus with $B$. ovis PA whole cells

Mice were immunized with $B$. melitensis Rev1, B. ovis $\Delta o m p 25 d$, B. ovis $\Delta$ omp 22 (retrospectively determined doses of $0.9 \times 10^{5}, 1.1 \times 10^{7}$, and $1.4 \times 10^{8} \mathrm{CFU} /$ mouse, respectively) or with PBS.

To evaluate the ability of spleen cells of the vaccinated animals to respond to a second stimulus by antigen,

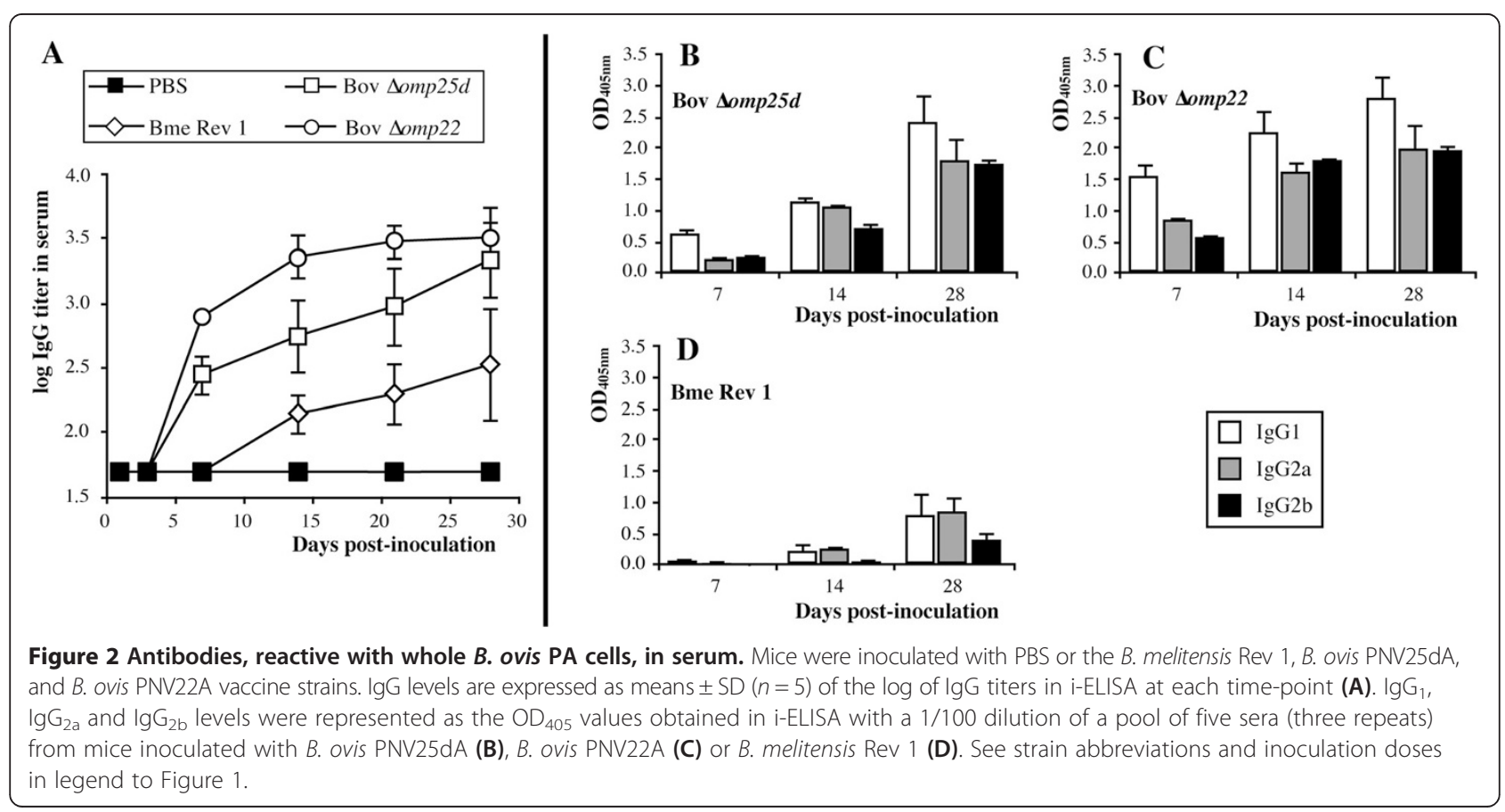



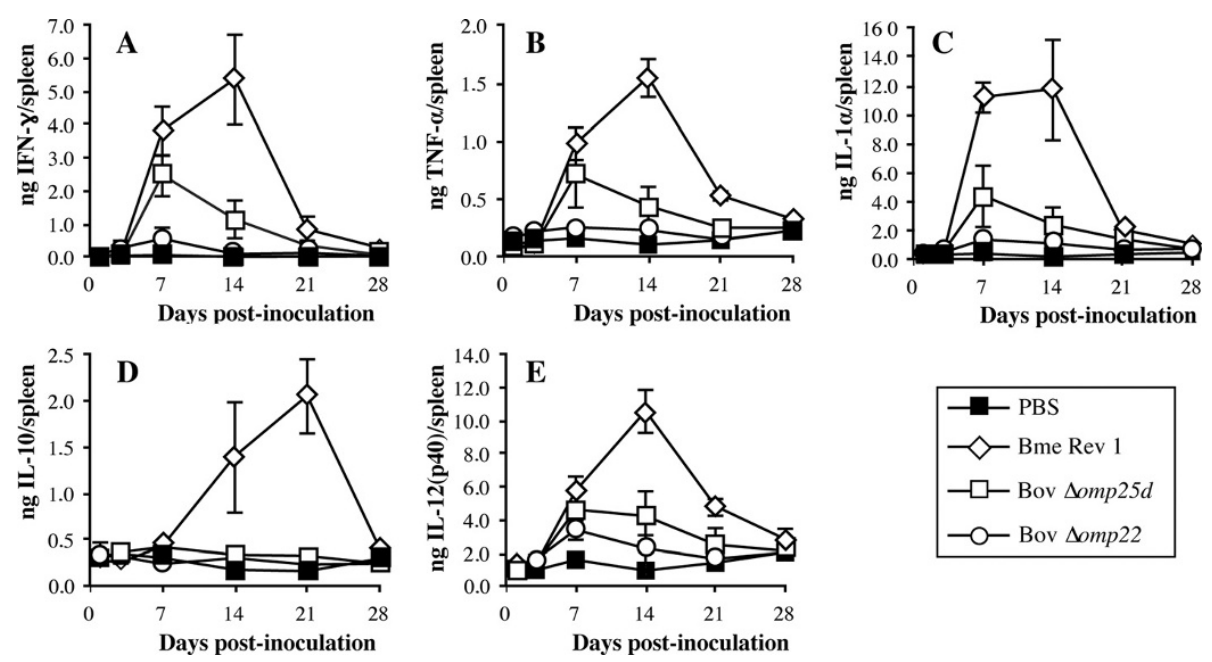

Figure 3 Levels of cytokines in spleen. IFN- $\gamma(\mathbf{A})$, TNF-a (B), IL-1a (C), IL-10 (D), and IL-12(p40) (E) were evaluated in the spleens of mice inoculated with PBS or the B. melitensis Rev 1, B. ovis PNV25dA, and B. ovis PNV22A vaccine strains. See strain abbreviations and inoculation doses in legend to Figure 1. Results are expressed as means \pm SD $(n=5)$ of each cytokine amount in spleen at each time-point.

splenocytes obtained on day 28 pi were stimulated with heat-inactivated B. ovis PA and the cytokines secreted were quantified after $72 \mathrm{~h}$ of incubation (see Materials and Methods).

Splenocytes from mice immunized with $B$. melitensis Rev 1 or with the two B. ovis attenuated mutants secreted high levels of IFN- $\gamma$ (ranging between 97 and $223 \mathrm{ng} /$ well), which was the most abundant cytokine, in response to the second antigen stimulus with B. ovis PA (Figure 4A). By contrast, IFN- $\gamma$ production by splenocytes obtained from mice inoculated with PBS was comparatively weak (about $5 \mathrm{ng} /$ well; $P<0.0005$ ) (Figure 4A).

The three attenuated vaccines induced the secretion of TNF- $\alpha$ by stimulated spleen cells to a similar extent and in amounts that were two times higher $(P<0.05)$ than those detected in the group inoculated with PBS (Figure 4B). Regarding the production of IL- $1 \alpha$, no differences were found between the stimulated splenocytes of mice immunized with B. melitensis Rev 1 and those of the control mice inoculated with PBS (Figure 4C). In contrast,
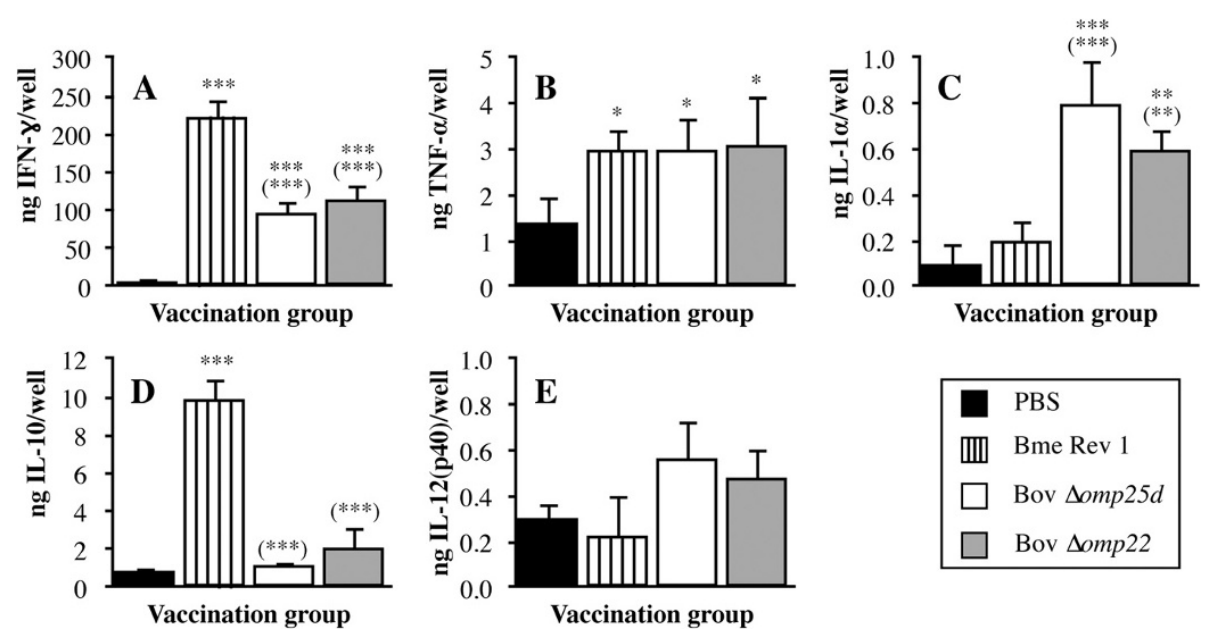

Figure 4 Cytokine production by stimulated splenocytes obtained from mice inoculated with PBS or the vaccine strains. Mice were inoculated with PBS or with B. melitensis Rev 1, B. ovis PNV25dA, and B. ovis PNV22A vaccine strains. Spleen cells were obtained 28 days after vaccine inoculation from five mice per group. Splenocytes were cultured as described in Materials and methods and stimulated with heat-killed $B$. ovis PA ( $10^{7}$ CFU/well) for $72 \mathrm{~h}$. Supernatants were analyzed by ELISA tests specific for IFN- $\gamma$ (A), TNF-a (B), IL-1a (C), IL-10 (D), and IL-12(p40) (E). Results are expressed as means \pm SD $(n=5)$ of each cytokine amount per well. See strain abbreviations and inoculation doses in legend to Figure 1. Significant differences (PLSD test) between mice inoculated with each vaccine and control mice inoculated with PBS are marked as follows: ${ }^{* *}, P \leq 0.0005$; ${ }^{* *}$, $P \leq 0.005 ; *, P \leq 0.05$. Significant differences between mice inoculated with the $B$. ovis attenuated strains and mice vaccinated with $B$. melitensis Rev 1 are represented in brackets following the same rule. 
the production of IL- $1 \alpha$ in the B. ovis $\Delta o m p 25 d$ and $B$. ovis $\Delta$ omp22 vaccination groups was about four times and three times higher, respectively (Figure 4C).

A strong IL-10 response was detected in stimulated spleen cells of mice immunized with the B. melitensis Rev 1 classic vaccine (Figure 4D), while the spleen cells of mice vaccinated with the two $B$. ovis attenuated strains behaved similarly to those obtained from mice immunized with PBS (Figure 4D). Regarding the secretion of IL-12 (p40) by stimulated spleen cells, no statistically significant differences were found between the mice immunized with the attenuated Brucella strains and the control mice immunized with PBS (Figure 4E).

\section{Protective efficacy of the attenuated mutants against}

\section{B. ovis infection}

Mice immunized with PBS (negative immunization control), B. melitensis Rev 1, B. ovis $\Delta$ omp 25 d or B. ovis $\Delta o m p 22$ (retrospectively determined doses of $0.9 \times 10^{5}$, $1.1 \times 10^{7}$, and $1.4 \times 10^{8} \mathrm{CFU} /$ mouse, respectively) were challenged 28 days after immunization with virulent $B$. ovis PA (retrospectively determined dose of $0.9 \times 10^{5}$ $\mathrm{CFU} /$ mouse). Bacteria were counted in spleen 3 weeks after the experimental infection.

The $B$. melitensis Rev 1 classic vaccine and the two $B$. ovis attenuated strains conferred similar protection $(P>0.05)$ against a challenge with $B$. ovis. When compared to mice inoculated with PBS, the three attenuated vaccines reduced spleen colonization of virulent $B$. ovis PA in the order of $3 \log$ units (Figure 5). Only the challenge strain was isolated in mice vaccinated with $B$. ovis $\Delta o m p 25 d$ while

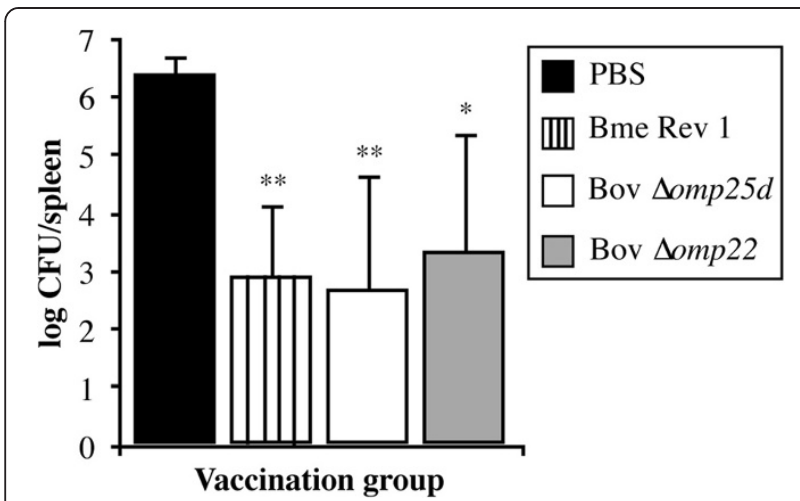

Figure 5 Protection conferred in mice against virulent $B$. ovis PA by the Brucella vaccines. Mice were inoculated with PBS (negative control), B. melitensis Rev 1, B. ovis PNV25dA, or B. ovis PNV22A (see strain abbreviations and inoculation doses in legend to Figure 1) and were challenged 28 days later with $10^{5} \mathrm{CFU} /$ mouse of virulent $B$. ovis PA. Three weeks after infection, the CFUs of B. ovis PA in spleen were counted in five mice per vaccination group. Results are expressed as means $\pm \mathrm{SD}(n=5)$ of the log CFU/spleen of $B$. ovis PA. Significant differences (PLSD test) between vaccination groups are marked as described in the legend to Figure 4. the B. ovis $\Delta o m p 22$ and B. melitensis Rev 1 vaccine strains were detected in 3 out 5 mice.

\section{Discussion}

$B$. melitensis Rev 1 is considered the best vaccine available against $B$. ovis infection but it has important drawbacks that constrain its use for the specific prophylaxis of brucellosis caused by B. ovis [3-7]. Since live attenuated vaccines have provided the best results in terms of protection against infections caused by other Brucella species [29-31], it is to be expected that an attenuated strain would also be the best choice for a vaccine targeting $B$. ovis infection. Smooth vaccine strains other than $B$. melitensis Rev 1 (i.e. B. abortus S19 or B. suis S2) would not avoid the interference with the serological diagnosis of smooth strain infections and, consequently, are not suitable vaccines. Theoretically, rough $B$. abortus RB51 would offer a good alternative because interference with diagnosis would be minimized. However, in spite of the rough surface phenotype of $B$. abortus RB51, the risk of inducing O-PS antibodies cannot be ignored owing to the presence of small amounts of O-PS in its cytoplasm [32] and, more importantly, this attenuated vaccine proved to be inefficient in preventing against $B$. ovis infection in rams [6]. Moreover, although some cases of heterologous protection have been reported, in livestock homologous Brucella spp. are usually more protective than heterologous Brucella spp. [31]. Accordingly, a B. ovis attenuated strain would likely be the most interesting candidate to develop a specific vaccine against ram contagious epididymitis. This hypothesis also seems to be supported by the fact that $B$. ovis displays significant differences in surface topology, outer membrane properties and antigenic behavior when compared to other Brucella species [2,21,33-35].

Although a recent work has reported the ability of a $B$. ovis attenuated mutant to induce an immune response in a natural host [36] and another work has analyzed the protective activity of a $B$. ovis mutant in a murine model [37], the potential of attenuated B. ovis strains as vaccines remains almost unexplored. Two $B$. ovis mutants defective in Omp25d and Omp22 surface protein, have previously been shown to display attenuated virulence in a mouse model $[19,38]$ and in the present work they were evaluated as homologous live attenuated vaccines, in comparison to heterologous $B$. melitensis $\operatorname{Rev} 1$, for the prophylaxis of $B$. ovis infection.

In a previous study, when inoculated at a dose of $5 \times$ $10^{6} \mathrm{CFU} / \mathrm{mouse}$ the $\Delta o m p 22$ mutant of $B$. ovis PA was almost undetectable in spleen from week 1 pi [19], while the $\Delta o m p 25 d$ mutant displayed a more moderate attenuation, with even higher spleen colonization during the initial stages of infection than that of the parental strain but with a rapid clearance thereafter [19]. However, 
when the immunization dose was increased to $1 \times 10^{8}$ $\mathrm{CFU} /$ mouse, even though maximum bacterial counts were $2 \log$ units lower than those of $B$. melitensis $\operatorname{Rev} 1$, the persistence in spleen of B. ovis $\Delta$ omp 22 was similar to that observed for the Rev 1 vaccine (Figure $1 \mathrm{~A}$ ) at the dose commonly used for protection studies in mouse models [22] and also in this work. The increase in the inoculation dose of the $\Delta$ omp $25 d$ mutant led to a spleen growth curve that exhibited the same pattern as that observed for the classic vaccine $B$. melitensis Rev 1 under our assay conditions of (Figure 1A). Accordingly, the requirement of a certain degree of persistence considered necessary for an efficacious vaccine against Brucella spp. $[39,40]$ seems to have been accomplished with the B. ovis $\Delta o m p 25 d$ and $\Delta o m p 22$ mutants, since their evolution in spleen was similar to that observed with a recognized control of vaccine efficacy against B. ovis (Figure 1A).

B. melitensis Rev 1 induced a strong inflammatory response in spleen, this response reaching its highest level of intensity on day $14 \mathrm{pi}$, while B. ovis $\Delta o m p 25 d$, with a spleen colonization profile similar to that of $B$. melitensis Rev 1 (Figure 1A), and B. ovis $\Delta o m p 22$ only elicited a moderate degree of splenomegaly (Figure 1B). Spleen inflammation in the mice inoculated with $B$. melitensis Rev 1 correlated with the high levels of cytokines detected in this organ, since with the exception of IL-10 all the cytokines peaked on day 14 pi (Figure 3), concomitantly with spleen weight (Figure 1B). In these mice, the maximum spleen levels of IL-10 were reached one week later (Figure 3D), which, considering the antiinflammatory role proposed for this cytokine, could reflect a mechanism aimed at counterbalancing the deleterious effects in the host derived from the exacerbated inflammatory response $[41,42]$. This hypothesis seems to be supported by the fact that the increase in IL-10 observed in mice vaccinated with $B$. melitensis Rev 1 (Figure 3D) was accompanied by a striking reduction in both spleen weight (Figure 1B) and the amount of the other cytokines analyzed (Figure 3 ). The reduction in cytokine levels continued until day 28 post-vaccination, when they reached the basal values observed in mice inoculated with $\mathrm{PBS}$ or with the $B$. ovis-derived vaccines (Figure 3). Since the challenge with virulent $B$. ovis PA was performed at that moment, the protection afforded by the vaccines cannot be attributed to residual cytokines from the primary immunization. However, vaccination with $B$. melitensis $\operatorname{Rev} 1$ or the attenuated $\Delta o m p 22$ and $\Delta o m p 25 d$ strains of $B$. ovis induced a memory immune response that could be activated by $B$. ovis cells and that was evidenced by the pattern of cytokine secretion when splenocytes obtained from vaccinated mice were re-stimulated ex vivo with killed B. ovis PA whole cells (Figure 4).

The strong inflammatory response -revealed by the prominent increase in spleen weight and cytokine levels- detected in the spleens of mice vaccinated with $B$. melitensis Rev 1 with respect to that induced by the $B$. ovis vaccines (Figures $1 \mathrm{~B}$ and 2 ) would initially predict a better protective activity for the former vaccine. However, the protection conferred by B. ovis $\Delta$ omp $25 d$ and $\Delta o m p 22$ against an experimental $B$. ovis infection was equivalent to that of B. melitensis Rev 1 (Figure 5), and several reasons can be invoked to explain this. First, the higher response of antibodies able to bind to $B$. ovis whole cells detected in mice immunized with the $B$. ovis-derived vaccines (Figure 2) constitutes an advantageous characteristic of the homologous vaccines over B. melitensis Rev 1 , since a more prominent role for antibodies than for $\mathrm{T}$ lymphocytes has been identified in protective immunity against B. ovis [43]. Second, the level of IFN- $\gamma$, which is known to play a critical role in the control of primary and secondary Brucella infections $[44,45]$, secreted by spleen cells from immunized mice in response to exposure to $B$. ovis whole cells was remarkably high (ranging between 97 and $223 \mathrm{ng} /$ well) in the three vaccination groups (Figure 4A). Third, splenocytes from mice vaccinated with the $B$. ovis-derived vaccines secreted more IL-1 $\alpha$, which has been shown to be a potent enhancer of CD4 and CD8 $\mathrm{T}$ cell responses to antigen [46], than splenocytes obtained from mice vaccinated with B. melitensis Rev 1 or non-vaccinated mice (Figure 4C). Finally, re-stimulated spleen cells of mice vaccinated with the $B$. ovis attenuated mutants did not differ from those obtained from non-vaccinated mice regarding the production of IL-10, while vaccination with $B$. melitensis Rev 1 induced an intense IL-10 response in re-stimulated splenocytes. Considering the anti-inflammatory role proposed for this cytokine [41,42], this might also constitute an advantage for the $B$. ovis attenuated vaccines.

Some of these valuable characteristics of B. ovis $\Delta$ omp $25 d$ and $\Delta o m p 22$ are probably related to a more specific immune response induced when homologous vaccines are used. Despite the antigenic differences described for $B$. ovis in comparison with other Brucella species, only a moderate degree of antigenic diversity is expected between B. melitensis Rev 1 and B. ovis PA, considering the high degree of DNA identity shared by them [47]. However, S-LPS is the major immunodominant antigen in smooth strains such as B. melitensis Rev 1 [48,49], while R-LPS and OMPs are immunodominant components in rough $B$. ovis that lack S-LPS [50]. Accordingly, an important percentage of the immune response developed after vaccination with $B$. melitensis Rev 1 would probably not be effective against later infection by B. ovis. By contrast, the immune response triggered by the $B$. ovis attenuated vaccines would target homologous antigens during a B. ovis infection and would theoretically be more efficient in controlling the disease. Another positive aspect of B. ovis $\triangle o m p 25 d$ and $\Delta o m p 22$ is that their protective activity is accompanied by a limited 
degree of splenomegaly, suggesting that these vaccines would prevent undesirable effects associated with inflammation in the host, as described for the classic vaccines B. abortus S19 and B. melitensis Rev 1 [51,52].

According to the results reported here, the attenuated strains B. ovis $\Delta$ omp $25 d$ and $\Delta o m p 22$ induce a solid immune response, with mixed Th1 and Th2 components, able to control later infection by $B$. ovis as efficiently as vaccination with heterologous $B$. melitensis Rev 1 . Considering the drawbacks of vaccination with $B$. melitensis $\operatorname{Rev} 1[5,8,9]$, especially for the prophylaxis of B. ovis infection, the attenuated vaccines analyzed here constitute a promising alternative as specific vaccines against ram contagious epididymitis that merits further evaluation in the natural host and the analysis of alternative formulations that might increase protective activity, such as microencapsulation for sustained release, as described for other Brucella vaccines [51,52].

\section{Competing interests}

The authors declare that they have no competing interests.

\section{Authors' contributions}

PS, CT, LFL and NV conceived the study and participated in its design and coordination. PS, RSSM, and NV performed the experiments. PS, CT, LFL and NV wrote the manuscript. All authors read and approved the final manuscript.

\section{Acknowledgments}

We thank Nicholas Skinner for supervising the English version of this manuscript and the staff of the animal experimentation facilities of the University of Salamanca for their helpful collaboration. Financial support to Rebeca S. Sidhu-Muñoz (FPI grant BES-2012-057056) and this work (reference projects AGL2008-04514-C03-02/GAN and AGL2011-30453-C04-02) was provided by the Ministerio de Economía y Competitividad of Spain.

Received: 30 March 2014 Accepted: 23 May 2014

Published: 4 June 2014

\section{References}

1. Burgess GW: Ovine contagious epididymitis: a review. Vet Microbiol 1982, 7:551-575

2. Vizcaíno N, Cloeckaert A: Biology and genetics of the Brucella outer membrane. In Brucella molecular microbiology and genomics. Edited by LópezGoñi I, O'Callaghan D. Norfolk, UK: Caister Academic Press; 2012:133-161.

3. OIE: Chapter 2.7.9. Ovine epididymitis (Brucella ovis). In Manual of diagnostic tests and vaccines for terrestrial animals; 2013. [http://www.oie.int/ en/international-standard-setting/terrestrial-manual/access-online/].

4. Blasco JM: Brucella ovis. In Animal brucellosis. Edited by Neilsen K, Duncan JR. Boca Raton, FL, USA: CRC Press; 1990:351-378.

5. Blasco JM: A review of the use of $B$. melitensis Rev 1 vaccine in adult sheep and goats. Prev Vet Med 1997, 31:275-283.

6. Jiménez de Bagüés MP, Barberán M, Marín CM, Blasco JM: The Brucella abortus RB51 vaccine does not confer protection against Brucella ovis in rams. Vaccine 1995, 13:301-304

7. Ridler AL, West DM: Control of Brucella ovis infection in sheep. Vet Clin North Am Food Anim Pract 2011, 27:61-66.

8. Alton GG, Jones LM, Angus RD, Verger JM: Techniques for the brucellosis laboratory. Paris, France: Institut National de la Recherche Agronomique; 1988.

9. Grilló MJ, Bosseray N, Blasco JM: In vitro markers and biological activity in mice of seed lot strains and commercial Brucella melitensis Rev 1 and Brucella abortus B19 vaccines. Biologicals 2000, 28:119-127.

10. Murillo M, Grilló MJ, Reñé J, Marín CM, Barberán M, Goñi MM, Blasco JM, Irache JM, Gamazo C: A Brucella ovis antigenic complex bearing poly-epsilon-caprolactone microparticles confer protection against experimental brucellosis in mice. Vaccine 2001, 19:4099-4106.
11. Blasco JM, Gamazo C, Winter AJ, Jiménez de Bagüés MP, Marín C, Barberán M, Moriyón I, Alonso-Urmeneta B, Díaz R: Evaluation of whole cell and subcellular vaccines against Brucella ovis in rams. Vet Immunol Immunopathol 1993, 37:257-270.

12. Da Costa Martins R, Irache JM, Blasco JM, Muñoz MP, Marín CM, Grilló MJ, De Miguel MJ, Barberán M, Gamazo C: Evaluation of particulate acellular vaccines against Brucella ovis infection in rams. Vaccine 2010, 28:3038-3046.

13. Estein SM, Fiorentino MA, Paolicchi FA, Clausse M, Manazza J, Cassataro J, Giambartolomei GH, Coria LM, Zylberman V, Fossati CA, Kjeken R, Goldbaum FA: The polymeric antigen BLSOmp31 confers protection against Brucella ovis infection in rams. Vaccine 2009, 27:6704-6711.

14. Estein SM, Cassataro J, Vizcaíno N, Zygmunt MS, Cloeckaert A, Bowden RA: The recombinant Omp31 from Brucella melitensis alone or associated with rough lipopolysaccharide induces protection against Brucella ovis infection in BALB/c mice. Microbes Infect 2003, 5:85-93.

15. Cassataro J, Pasquevich KA, Estein SM, Laplagne DA, Zwerdling A, de la Barrera S, Bowden R, Fossati CA, Giambartolomei GH, Goldbaum FA: A DNA vaccine coding for the chimera BLSOmp31 induced a better degree of protection against $B$. ovis and a similar degree of protection against $B$. melitensis than Rev.1 vaccination. Vaccine 2007, 25:5958-5967.

16. Cassataro J, Velikovsky CA, de la Barrera S, Estein SM, Bruno L, Bowden R, Pasquevich KA, Fossati CA, Giambartolomei GH: A DNA vaccine coding for the Brucella outer membrane protein 31 confers protection against $B$. melitensis and $B$. ovis infection by eliciting a specific cytotoxic response. Infect Immun 2005, 73:6537-6546.

17. Estein SM, Cheves PC, Fiorentino MA, Cassataro J, Paolicchi FA, Bowden RA: Immunogenicity of recombinant Omp31 from Brucella melitensis in rams and serum bactericidal activity against B. ovis. Vet Microbiol 2004, 102:203-213.

18. Nicoletti P: Brucellosis: past, present and future. Prilozi 2010, 31:21-32.

19. Caro-Hernández P, Fernández-Lago L, de Miguel MJ, Martín-Martín Al, Cloeckaert A, Grilló MJ, Vizcaíno N: Role of the Omp25/Omp31 family in outer membrane properties and virulence of Brucella ovis. Infect Immun 2007, 75:4050-4061.

20. Salhi I, Boigegrain RA, Machold J, Weise C, Cloeckaert A, Rouot B: Characterization of new members of the group 3 outer membrane protein family of Brucella spp. Infect Immun 2003, 71:4326-4332.

21. Vizcaíno N, Caro-Hernández P, Cloeckaert A, Fernández-Lago L: DNA polymorphism in the omp25/omp31 family of Brucella spp.: identification of a 1.7-kb inversion in Brucella cetaceae and of a 15.1-kb genomic island, absent from Brucella ovis, related to the synthesis of smooth lipopolysaccharide. Microbes Infect 2004, 6:821-834.

22. Grilló MJ, Blasco JM, Gorvel JP, Moriyón I, Moreno E: What have we learned from brucellosis in the mouse model? Vet Res 2012, 43:29.

23. OIE: Chapter 2.7.2. Caprine and ovine brucellosis (excluding Brucella ovis). In Manual of Diagnostic Tests and Vaccines for Terrestrial Animals; 2013. [http://www.oie.int/eng/normes/mmanual/a_summry.htm].

24. Silva TM, Costa EA, Paixão TA, Tsolis RM, Santos RL: Laboratory animal models for brucellosis research. J Biomed Biotechnol 2011, 2011:518323.

25. Barrio MB, Grilló MJ, Muñoz PM, Jacques I, González D, de Miguel MJ, Marín CM, Barberán M, Letesson JJ, Gorvel JP, Moriyón I, Blasco JM, Zygmunt MS: Rough mutants defective in core and O-polysaccharide synthesis and export induce antibodies reacting in an indirect ELISA with smooth lipopolysaccharide and are less effective than Rev 1 vaccine against Brucella melitensis infection of sheep. Vaccine 2009, 27:1741-1749.

26. Fernández-Lago L, Monte M, Chordi A: Endogenous gamma interferon and interleukin-10 in Brucella abortus 2308 infection in mice. FEMS Immunol Med Microbiol 1996, 15:109-114.

27. Fernández-Lago L, Rodríguez-Tarazona E, Vizcaíno N: Differential secretion of interleukin-12 (IL-12) subunits and heterodimeric IL-12p70 protein by CD-1 mice and murine macrophages in response to intracellular infection by Brucella abortus. J Med Microbiol 1999, 48:1065-1073.

28. Fernández-Lago L, Orduña A, Vizcaíno N: Reduced interleukin-18 secretion in Brucella abortus 2308-infected murine peritoneal macrophages and in spleen cells obtained from B. abortus 2308-infected mice. J Med Microbiol 2005, 54:527-531.

29. Godfroid J, Scholz HC, Barbier T, Nicolas C, Wattiau P, Fretin D, Whatmore AM, Cloeckaert A, Blasco JM, Moriyón I, Saegerman C, Muma JB, Al Dahouk $\mathrm{S}$, Neubauer H, Letesson JJ: Brucellosis at the animal/ecosystem/human interface at the beginning of the 21st century. Prev Vet Med 2011, 102:118-131. 
30. Ficht TA, Kahl-McDonagh MM, Arenas-Gamboa AM, Rice-Ficht AC: Brucellosis: the case for live, attenuated vaccines. Vaccine 2009, 27(Suppl 4):D40-D43.

31. Adams LG: Development of live Brucella vaccines. In Advances in brucellosis research. Edited by Adams LG. College Station. USA: Texas A\&M University Press; 1990.

32. Cloeckaert A, Zygmunt MS, Guilloteau LA: Brucella abortus vaccine strain RB51 produces low levels of M-like O-antigen. Vaccine 2002, 20:1820-1822.

33. Cloeckaert A, Verger JM, Grayon M, Zygmunt MS, Grépinet O: Nucleotide sequence and expression of the gene encoding the major 25-kilodalton outer membrane protein of Brucella ovis: Evidence for antigenic shift, compared with other Brucella species, due to a deletion in the gene. Infect Immun 1996, 64:2047-2055.

34. Martín-Martín Al, Sancho P, Tejedor C, Fernández-Lago L, Vizcaíno N: Differences in the outer membrane-related properties of the six classical Brucella species. Vet J 2011, 189:103-105.

35. Vizcaíno N, Kittelberger R, Cloeckaert A, Marín CM, Fernández-Lago L: Minor nucleotide substitutions in the omp31 gene of Brucella ovis result in antigenic differences in the major outer membrane protein that it encodes compared to those of the other Brucella species. Infect Immun 2001, 69:7020-7028.

36. Silva AP, Macêdo AA, Costa LF, Turchetti AP, Bull V, Pessoa MS, Araújo MS, Nascimento EF, Martins-Filho OA, Paixão TA, Santos RL: Brucella ovis lacking a species-specific putative ATP-binding cassette transporter is attenuated but immunogenic in rams. Vet Microbiol 2013, 167:546-553.

37. Edmonds MD, Cloeckaert A, Elzer PH: Brucella species lacking the major outer membrane protein Omp25 are attenuated in mice and protect against Brucella melitensis and Brucella ovis. Vet Microbiol 2002, 88:205-221.

38. Martín-Martín Al, Caro-Hernández P, Orduña A, Vizcaíno N, Fernández-Lago $\mathrm{L}$ : Importance of the Omp25/Omp31 family in the internalization and intracellular replication of virulent $B$. ovis in murine macrophages and HeLa cells. Microbes Infect 2008, 10:706-710

39. Kahl-McDonagh MM, Ficht TA: Evaluation of protection afforded by Brucella abortus and Brucella melitensis unmarked deletion mutants exhibiting different rates of clearance in BALB/c mice. Infect Immun 2006, 74:4048-4057

40. Ko J, Splitter GA: Molecular host-pathogen interaction in brucellosis: current understanding and future approaches to vaccine development for mice and humans. Clin Microbiol Rev 2003, 16:65-78.

41. Rutz S, Ouyang W: Regulation of interleukin-10 and interleukin-22 expression in T helper cells. Curr Opin Immunol 2011, 23:605-612

42. Corsetti PP, de Almeida LA, Carvalho NB, Azevedo V, Silva TM, Teixeira HC, Faria AC, Oliveira SC: Lack of endogenous IL-10 enhances production of proinflammatory cytokines and leads to Brucella abortus clearance in mice. PLoS One 2013, 8:e74729.

43. Jiménez de Bagüés MP, Elzer PH, Blasco JM, Marín CM, Gamazo C, Winter AJ: Protective immunity to Brucella ovis in BALB/c mice following recovery from primary infection or immunization with subcellular vaccines. Infect Immun 1994, 62:632-638.

44. Zhan Y, Cheers C: Endogenous gamma interferon mediates resistance to Brucella abortus infection. Infect Immun 1993, 61:4899-4901.

45. Murphy EA, Sathiyaseelan J, Parent MA, Zou B, Baldwin CL: Interferon-gamma is crucial for surviving a Brucella abortus infection in both resistant C57BL/6 and susceptible BALB/c mice. Immunology 2001, 103:511-518.

46. Ben-Sasson SZ, Caucheteux S, Crank M, Hu-Li J, Paul WE: IL-1 acts on T cells to enhance the magnitude of in vivo immune responses. Cytokine 2011 56:122-125.

47. Verger JM, Grimont F, Grimont PA, Grayon M: Brucella, a monospecific genus as shown by deoxyribonucleic-acid hybridization. Int J Syst Bacteriol 1985, 35:292-295

48. Wright PF, Nielsen $\mathrm{KH}$ : Current and future serological methods. In Advances in brucellosis research. Edited by Adams LG. College Station. USA: Texas A\&M University Press; 1990.

49. Zygmunt MS, Debbarh HS, Cloeckaert A, Dubray G: Antibody response to Brucella melitensis outer membrane antigens in naturally infected and Rev1 vaccinated sheep. Vet Microbiol 1994, 39:33-46.

50. Kittelberger R, Hilbink F, Hansen MF, Ross GP, de Lisle GW, Cloeckaert A, de Bruyn J: Identification and characterization of immunodominant antigens during the course of infection with Brucella ovis. J Vet Diagn Invest 1995, 7:210-218.

51. Arenas-Gamboa AM, Ficht TA, Kahl-McDonagh MM, Gomez G, Rice-Ficht AC The Brucella abortus S19 $\Delta v j b R$ live vaccine candidate is safer than S19 and confers protection against wild-type challenge in BALB/c mice when delivered in a sustained-release vehicle. Infect Immun 2009, 77:877-884.

52. Arenas-Gamboa AM, Ficht TA, Kahl-McDonagh MM, Rice-Ficht AC: Immunization with a single dose of a microencapsulated Brucella melitensis mutant enhances protection against wild-type challenge. Infect Immun 2008, 76:2448-2455.

doi:10.1186/1297-9716-45-61

Cite this article as: Sancho et al:: Evaluation in mice of Brucella ovis attenuated mutants for use as live vaccines against $B$. ovis infection. Veterinary Research 2014 45:61.

\section{Submit your next manuscript to BioMed Central and take full advantage of:}

- Convenient online submission

- Thorough peer review

- No space constraints or color figure charges

- Immediate publication on acceptance

- Inclusion in PubMed, CAS, Scopus and Google Scholar

- Research which is freely available for redistribution

Submit your manuscript at www.biomedcentral.com/submit
C Biomed Central 